\title{
Abnormal Motion Areas Detection for Advanced Driver Assistance System
}

\author{
Jianping Chen ${ }^{a}$, Husheng Liao, Lihua Fu \\ Beijing University of Technology, Beijing, China. \\ achentravelling@emails.bjut.edu.cn
}

Keywords: abnormal motion; abnormality; MMM; AMAD-ADAS; Reinhard.

\begin{abstract}
This paper proposes a novel abnormal motion detection method for advanced driver assistance system (AMAD-ADAS) containing techniques of lane markings detection, motion areas detection and abnormality quantification model. First, lane markings are detected based on Hough transform. Later, a novel motion areas detection method consisting of multiple layers operation, multiple motions operation and multiple areas operation (termed MMM method) is approved to detect candidate motion areas, which is more suitable for real environment than the traditional appearance-based method that can only detect specified object, such as vehicles and pedestrians. Finally, an abnormality quantification model is estimated to quantify the abnormality of each candidate area obtained by MMM method. The AMAD-ADAS shows its robustness through many experiments on the Reinhard [1] datasets.
\end{abstract}

\section{Introduction}

Recently, many researches of ADAS are proposed to assist drivers. The most important tasks in ADAS are object detection and the model to judge whether the object is a threat to ego vehicle. Current research methods of object detection have a remarkable production and application with the continuous and great development of computer vision technology. But most of these are based on appearance of images to detect object. That means they can only detect specified object which is trained. Actually, any object may be in the roadway and pose a threat to the driver.

The AMAD-ADAS approved in this paper is based on motion feature and contains lane marking detection, motion areas detection and abnormality quantification. If the abnormality of a motion area is bigger than a considered threshold, AMAD-ADAS sends a warning signal to driver and marks the abnormal area.

This paper is organized as follows: Section 2 describes the proposed AMAD-ADAS including lane markings detection, the MMM method and abnormality quantification model. The experimental results showing the robustness of AMAD-ADAS are displayed in section 3 and section 4 presents the acknowledgments.

\section{Proposed System}

The flowchart of AMAD-ADAS shown in Fig.1 is organized into three main components: lane markings detection, motion areas detection and abnormality quantification model. The system procures images from the video sequences. After pre-procession, the flowchart is split into two parts: lane markings detection and motion area detection. Finally, the process is merged into abnormality quantification model.

The three components are discussed in the following three sections respectively.

\section{Lane Markings Detection}

Lane markings play an important role in a real driving environment. This paper detects lane markings based on Hough transform [2] as follows. 


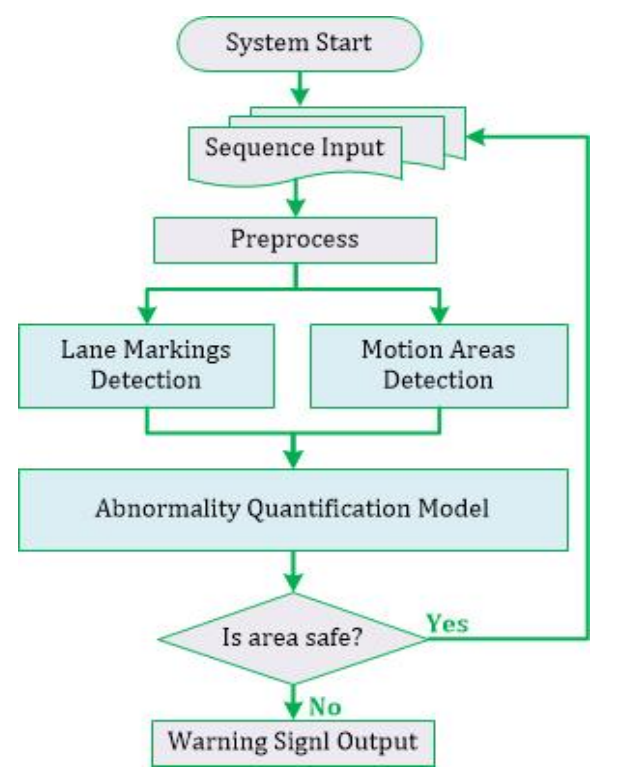

(a)

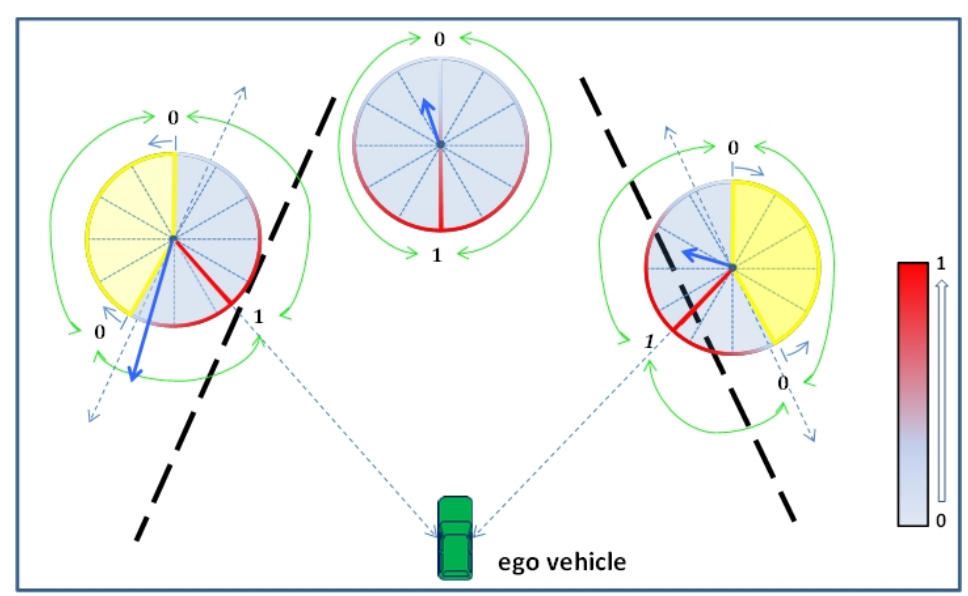

(b)

Figure 1. (a) The Flowchart of AMAD-ADAS Including Three Main Parts which are Lane Markings Detection, Abnormal Motion Areas Detection and Abnormality Quantification Model.

(b) The Diagram of the Weight Model of Motion Direction Contains Three Situations, which are the Area is in Left Lane, the Area is in Right Lane or in the Same Road with Vehicle.

Firstly, image is processed with binarization. Erode and Dilate algorithms are used to remove the noise and fill the holes and the edge features points are extracted based on Canny. Lastly, the lanes are selected from the candidate lines which are sifted out using Hough transform.

If there are no detected lane markings in the current frame, the lane markings detected in the previous frame will be used.

\section{Motion Areas Detection}

Motion areas detection is the key step in the system. This paper proposed a novel method to detect motion areas, which is termed MMM algorithm. Fig.2 shows the method flowchart including three main parts: multiple layers operation, multiple motions operation, multiple areas operation and each of them is discussed in detail below. This method is based on the optical flow and disparity which represents the motion information and spatial information respectively.

1). Calculation of Optical Flow and Disparity: There is a problem that the object without significant features may be missed using traditional feature point extraction method. Therefore, a set of pixels extracted from the ROI at a certain step length is used as the feature points in this paper. But, this method makes higher false matching rates in optical flow calculation using the L-K algorithm. In order to resolve this problem, the forward-backward error removal method [3] is used, which calculates motion vector in both directions, forward and backward. As a result, the set of feature point pairs $S_{t}=\left\{P_{t}, Q_{t}\right\}$ is obtained.

Meanwhile, the estimation of the distance between ego vehicle and the object is an important technique of the ADAS. The relationship [4] shown in equation (1) between disparity and distance in 3D stereo vision-based model is an effective means to get the real distance of object.

$$
d(x, y)=\left\{\begin{array}{c}
\frac{f * b}{\Delta(x, y)}, \Delta(x, y)>0 \\
0, \text { otherwise }
\end{array}\right.
$$

Where $d(x, y)$ is the real distance, $f$ is the focal length of the camera, $b$ is the baseline of both camera rigs, $\Delta(x, y)$ is the disparity of coordinate $(x, y)$. 
2). MMM method: This paper proposes a novel abnormal motion area detection method termed MMM method as shown in Fig.2.

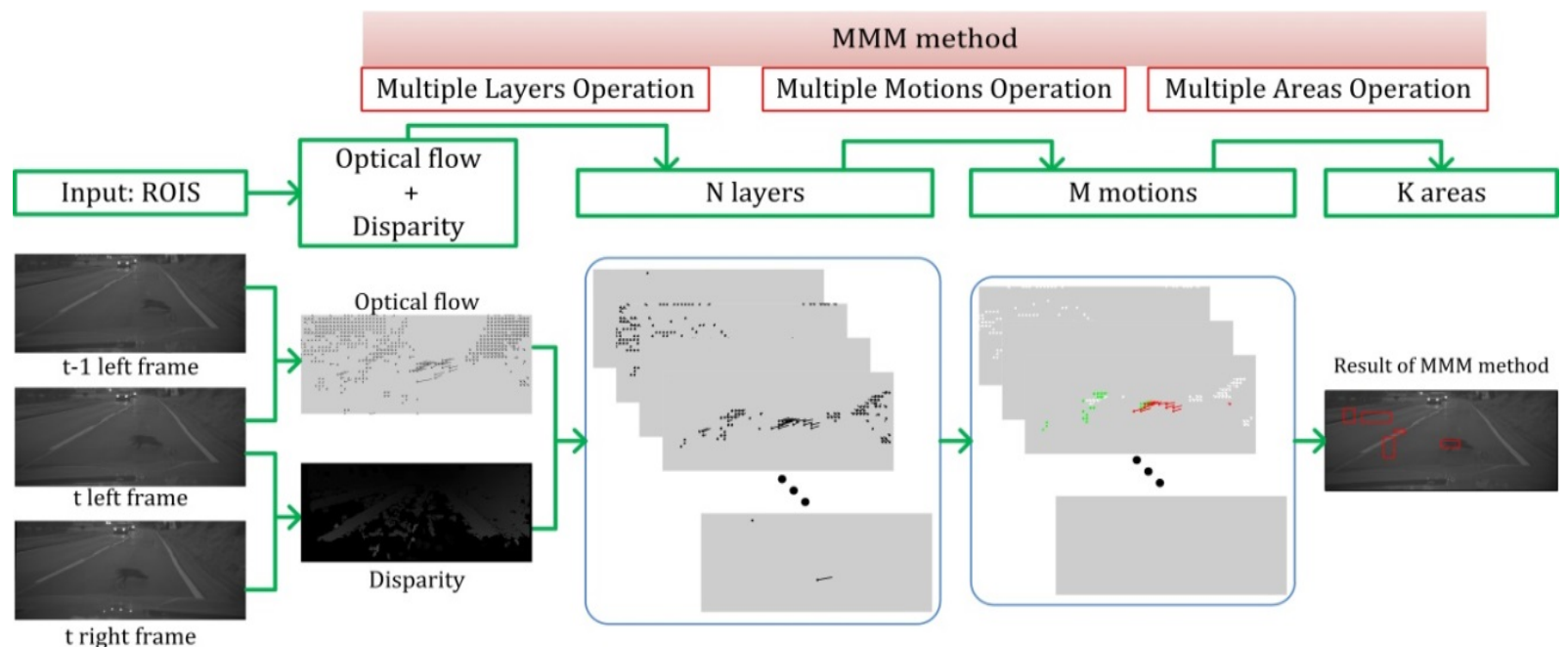

Figure 2. The Flowchart of MMM Method Based on Optical Flow and Disparity Contains Three Parts which are Multiple Layers Operation, Multiple Motions Operation and Multiple Areas Operation.

The ego motion of vehicle causes a great effect on moving object detection. Obviously, the effect is different on objects with different distance. This common sense gives the study the first inspiration to approve the multiple layers operation to deal with the feature points.

The distances of the feature points are calculated by equation (1), and then all points are sliced into $N$ layers $L_{\mathrm{t}}=\left\{L_{t}^{1}, L_{t}^{2}, \ldots, L_{t}^{N}\right\}$ on the basis of their distances, where $L_{t}^{l}=\left\{P_{t}^{l}, Q_{t}^{l}\right\}, l \in[1, N]$. Each layer represents a distance range. The multiple motions operation estimates several motion models for each layer by using the RANSAC algorithm several times. The distances of the points in the first layer are not bigger than zero, this is because the disparity values of the points do not exist or equal to zero. As a result, the first layer is ignored.

After multiple motions operation, $I_{t}^{l}$ formed $M$ inline point sets $\left\{I_{t}^{l, 1}, I_{t}^{l, 2}, \ldots, I_{t}^{l, m}\right\}$ are obtained, where $I_{t}^{l, m}=\left\{P_{t}^{l, m}, Q_{t}^{l, m}\right\}, m \in[1, M]$ stands for the number of set, and one motion model for one inline point set.

The next pivotal step is how to get the motion areas from the inline point sets. This paper approves the multiple areas operation based on DBSCAN algorithm to cluster each inline point set $I_{t}^{l, m}$ and calculate the abnormal feature vector of the area, which is discussed below.

There are two important parameters in DBSCAN, $R_{D B}$ and $N_{D B}$. The former represents the search radius and the latter represents the min number of points that can form a cluster. The two parameters are adjustable in this paper to make a better clustering result. At the same time, further screening the clustering results to remove improper areas is necessary and a feature vector is set for each area:

$$
v_{k}=\left(X_{\min }^{k}, Y_{\min }^{k}, X_{\text {max }}^{k}, Y_{\text {max }}^{k}, S_{k}, D_{k}, O_{k}, R_{k}\right)
$$

Where $\left(X_{\text {min }}^{k}, Y_{\text {min }}^{k}\right)$ and $\left(X_{\text {max }}^{k}, Y_{\text {max }}^{k}\right)$ are the coordinates of the top left point and bottom right point of area $v_{k}$ respectively. $S_{k}, D_{k}, O_{k}, R_{k}$ represents the motion size, distance to ego vehicle, motion orientation as well as the lane of the area $v_{k}$ respectively.

The motion size and direction of each point pairs in $v_{k}$ are defined in equation (3).This paper selects the motion size that contains the most point pairs as the $S_{k}$ of $v_{k}$ based on the histogram of motion size and the motion orientation which contains the most points is picked as the $O_{k}$ of $v_{k}$ based on rose diagram which is divided into $N_{B i n}$ areas. 


$$
\begin{gathered}
S(p)=\|p, q\| \\
O(p)=\operatorname{Tran}(\operatorname{atan} 2(q . y-p \cdot y, p \cdot x-q \cdot x)) * \frac{N_{B i n}}{2 \pi}
\end{gathered}
$$

Where $p, q$ are from one-point pair and function $\operatorname{Tran}(*)$ is turning $*$ to $[0,2 \pi]$.

The $D_{k}$ of $v_{k}$ is defined:

$$
\begin{gathered}
D_{k}=\frac{f^{*} b}{\Delta_{\text {ave }}^{k}} \\
\Delta_{\text {ave }}^{k}=\frac{1}{n} \sum_{X_{\min }^{k}}^{X_{\max }^{k}} \sum_{Y_{\min }^{k}}^{Y_{\max }^{k}} \Delta(i, j)
\end{gathered}
$$

Where $\Delta(i, j)$ is the disparity value of coordinate $(i, j)$ which is bigger than zero and $n$ is the number.

The calculation $R_{k}$ is based on the geometric relationship between the geometric center of $v_{k}$ and the lane markings. The value of $R_{k}$ is $0,1,2$ representing $v_{k}$ is on the left road, on the right road and on the same road with ego vehicle.

\section{Abnormality Quantification Model}

In this section, the Abnormality Quantification Model (AQM) is detailed that is approved for quantifying the abnormality of candidate areas. The abnormal feature vector of area $v_{k}$ is defined as equation (2).

This paper defines the motion size as the base parameter of abnormality, so the first step is calculating base abnormality caused by motion size:

$$
A_{b}^{k}=\frac{S_{k}-S_{\min }}{S_{\max }-S_{\min }}
$$

Where $S_{\min }, S_{\max }$ are the minimum and maximum motion sizes of the areas in image.

Considering the effect of distance on the abnormality, this paper supposes that the weight of distance $\omega_{k}$ of area $v_{k}$ follows Gauss distribution as shown in equation (6) and the exponential weighted operator shown in equation (7) is used to further describe the relationship between the distance and the weight, only when the distance is close enough that the weight will be strengthened, if not, the weight will be restrained.

$$
\begin{gathered}
\omega_{k}=e^{-\frac{\left(D_{k} / 10\right)^{2}}{2 \sigma^{2}}} \\
W_{D}^{k}=\left\{\begin{array}{c}
\left(1-e_{d}\right) *\left(\frac{\omega_{k}-e_{d}}{1-e_{d}}\right)^{n}+e_{d}, \omega_{k}>e_{d} \\
e_{d}-e_{d} *\left(\frac{e_{d}-\omega_{k}}{e_{d}}\right)^{n}, \omega_{k}<e_{d} \\
e_{d}, \omega_{k}=e_{d}
\end{array}\right.
\end{gathered}
$$

Where $e_{d}$ is the threshold of $\omega_{k}$ and $n=\ln 2 /\left(\ln 2-\ln \left(1-\partial_{d}\right)\right), \partial_{d}$ stands for the effect degree with a positive correlation, $W_{D}^{k}$ is the final weight value of distance $D_{k}$ for $v_{k}$.

The weight model of motion orientation is shown in Fig.1 (b). There are different solutions for different situations. In the model, the blue regions represent the range of motion directions used to compute the weights of motion orientation $W_{O}^{k}$ of $v_{k}$ which are continuous and $W_{O}^{k}$ in the yellow areas are zero.

Finally, the abnormality is defined:

$$
\Gamma=\operatorname{norm}\left(\operatorname{norm}\left(A_{b}^{k} * W_{D}^{k}\right) * W_{O}^{k}\right)
$$


Where the function norm $(*)$ is normalizing $*$ to $[0,1]$. If the abnormality value of object is bigger than an appropriate threshold, it should be marked and the system sends a warning message to the driver.

\section{Experimental Results}

To validate the AMAD-ADAS, sequence Squirrel in Reinhard is used in this paper, which shows a dangerous situation for drivers where a squirrel suddenly bursts into the roadway.

The experimental results are shown in Fig.3. There are three examples marked as (a)-(c) and each example contains four columns.

(a)
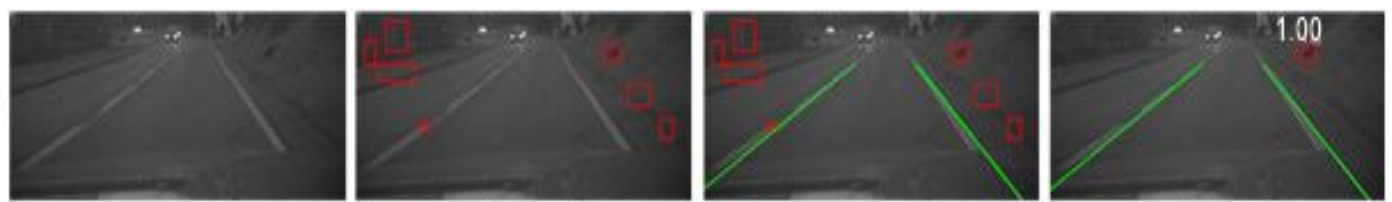

(b)
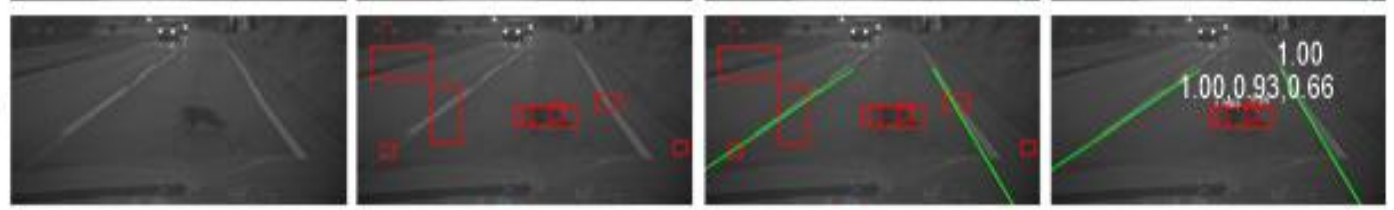

(c)
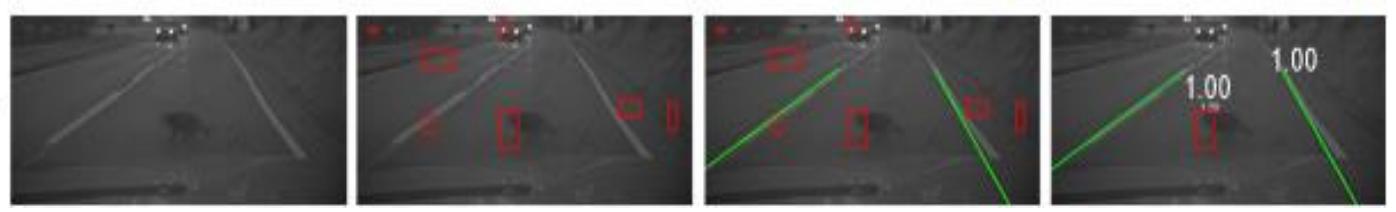

Figure 3. Some Example of Experimental Results of Sequence Squirrel

The ROI of input image are displayed in the first column. The abnormal areas are shown in the second column, which are detected by MMM method with $N=7$ in multiple layers operation and $M=4$ in multiple motions operation. The both detected lane markings and these areas are immediately followed. The final abnormal areas which are obtained by abnormality quantification model with $T_{D}$ and the threshold of $\Gamma$ is 0.6 is shown in the fourth column. In the first examples, the distant squirrel is on the hillside and running to the lane. In the example (b) and (c), the squirrel is on the same road with vehicle and running quickly. These example results demonstrate that this system not only detects abnormal areas within the road, also detects these staying outside the road.

\section{Acknowledgments}

This paper proposes an abnormal motion areas detection method for ADAS (AMAD-ADAS) which is based on motion feature and is more suitable for real environment than the traditional appearance-based method. The AMAD-ADAS shows its robustness through many experiments.

\section{References}

[1]. http://ccv.wordpress.fos.auckland.ac.nz.

[2]. Gaikwad V, Lokhande S. Lane Departure Identification for Advanced Driver Assistance J]. IEEE Transactions on Intelligent Transportation Systems, 2015, 16(2):910-918.

[3]. Kalal Z, Mikolajczyk K, Matas J. Forward-Backward Error: Automatic Detection of Tracking Failures. International Conference on Pattern Recognition. IEEE Computer Society, 2010:2756-2759.

[4]. Iloie A, Giosan I, Nedevschi S. UV disparity-based obstacle detection and pedestrian classification in urban traffic scenarios. IEEE International Conference on Intelligent Computer Communication and Processing. IEEE, 2014:119-125. 